\title{
Algas planctônicas do reservatório da Pampulha (MG): Euglenophyta, Chrysophyta, Pymophyta, Cyanobacteria
}

\author{
ALESSANDRA GIANI ${ }^{1}$, CLEBER C. FIGUEREDO $^{1}$ e PAULA C. ETEROVICK ${ }^{1}$
}

(recebido em 03/10/97; aceito em 13/11/98)

\begin{abstract}
Planktonic algae from Pampulha reservoir: Euglenophyta, Chrysophyta, Pyrrophyta, Cyanobacteria). This paper contains descriptions of 28 taxa of planktonic algae distributed in four divisions (Euglenophyta, Chrysophyta, Pyrrophyta and Cyanobacteria) found in the Pampulha reservoir in Minas Gerais state. Samples from May/1992 to February/1997 were analyzed. The best represented division was Euglenophyta (13 species in one family), followed by Cyanobacteria (eight species), Pyrrophyta (five species) and Chrysophyta (two species). Twenty three taxa are reported for the first time to the State of Minas Gerais.
\end{abstract}

RESUMO - (Algas planctônicas do reservatório da Pampulha (MG): Euglenophyta, Chrysophyta, Pyrrophyta, Cyanobacteria). Neste trabalho foram descritos 28 táxons de algas planctônicas pertencentes às divisões Euglenophyta, Chrysophyta, Pyrrophyta e Cyanobacteria, encontradas no reservatório da Pampulha, estado de Minas Gerais. Foram analisadas amostras no período de maio/1992 a fevereiro/ 1997. Em número de espécies, Euglenophyta foi a que apresentou maior número (13 espécies em uma família), seguidas de Cyanobacteria (oito espécies), Pyrrophyta (cinco espécies) e Chrysophyta (duas espécies). Vinte e três táxons são primeiras citações para o estado de Minas Gerais.

Key words - Taxonomy, Euglenophyta, Chrysophyta, Pyrrophyta, Cyanobacteria

\section{Introdução}

Este trabalho pretende dar continuidade ao estudo de algas planctônicas do reservatório da Pampulha, reservatório hipereutrófico situado na cidade de Belo Horizonte (MG). Em estudo anterior (Eterovick \& Giani 1997), foram descritas algas da divisão Chlorophyta neste mesmo ambiente e já se enfatizava a escassez de estudos ficoflorísticos no estado de Minas Gerais, apesar da sua grande riqueza e diversidade de ecossistemas aquáticos.

Pela sua localização urbana, pelo avançado estágio de degradação e pela ênfase que tem sido dada recentemente ao manejo e recuperação das suas águas, o reservatório da Pampulha tem atraído pesquisadores para realização de diversos estudos limnológicos (Pinto-Coelho et al. 1994, Araujo 1995, Goodwin 1997, entre outros). O conhecimento da ficoflórula, no seu aspecto taxonômico, torna-se importante base para desenvolvimento da maioria destes estudos.

Euglenofíceas pigmentadas são conhecidas por serem um grupo que se desenvolve principalmente em águas com altos teores de matéria orgânica, fósfo-

1. Departamento de Botânica, Instituto de Ciências Biológicas, Universidade Federal de Minas Gerais, Caixa Postal 486 31270-110 Belo Horizonte, MG, Brasil. E-mail: agiani@mono.icb.ufmg.br ro e nitrogênio (Alves-da-Silva \& Laitano 1994). Algas flageladas em geral, incluindo as dinofíceas, as criptofíceas e algumas crisofíceas, costumam apresentar elevadas biomassas em reservatórios hipereutróficos (Rojo \& Alvarez-Cobelas 1993). $\mathrm{O}$ fato de poderem se movimentar é uma vantagem em ambientes túrbidos com relação a luz e permite, ainda, que estas algas possam utilizar nutrientes presentes em camadas mais profundas, podendo em seguida voltar para a região eufótica. Além disso, o tipo de alimentação fagotrófica, que um grande número de flagelados apresenta (Porter 1989), é mais uma fonte de nutrição, nestes ambientes tão ricos em matéria orgânica. As cianobactérias, principalmente do grupo Microcystis, têm um papel ecológico muito importante em corpos d'água eutróficos, pela sua capacidade de se desenvolverem maciçamente ("water blooms") e pela sua potencial toxicidade. Apesar da sua importância e ampla distribuição, esses organismos apresentam uma taxonomia difícil, com a ocorrência comum das chamadas populações de "transição" (Komárek 1991), que parecem apresentar características de mais de uma espécie.

O objetivo deste trabalho foi descrever as principais algas planctônicas pertencentes às divisões Euglenophyta, Chrysophyta, Pyrrophyta e Cyanobacteria, comumente encontradas nesse reservatório. 


\section{Material e métodos}

As amostras foram coletadas mensalmente ou quinzenalmente durante o período de maio/1992 a fevereiro/1997, em estação única no reservatório da Pampulha (Belo Horizonte, MG), com redes de plâncton de $20 \mu \mathrm{m}$ de abertura de malha. Dados do local de coleta encontram-se em Eterovick \& Giani (1997). As amostras foram fixadas com solução de lugol acético ou de Transeau e armazenadas em frascos de vidro ao abrigo da luz. Amostras de material vivo foram observadas para detalhes morfológicos de algumas espécies.

As análises e identificações foram realizadas através de uso de microscópio Olympus com câmara clara e ocular micrometrada. Sempre que possível, foram observados diversos indivíduos de uma mesma espécie, para ressaltar o caráter populacional. Para a identificação dos táxons seguiram-se: Smith (1920), Deflandre (1926), Schiller (1937), Pochmann (1942), Balech (1944), Conrad \& Van Meel (1952), Desikachary (1959), Huber-Pestalozzi (1962, 1969), Parra et al. (1982), Anagnostidis \& Komárek (1988), Xavier (1989), Castro et al. (1991), Komárek (1991), KomárkóvaLegnerová \& Eloranta (1992), Rojo \& Alvarez-Cobelas (1993), Alves-da-Silva \& Laitano (1994), Menezes (1994) e Sant'Anna \& Azevedo (1995).

As análises taxonômicas apresentadas referem-se a 11 amostras selecionadas dentre as coletadas no período de estudo. As amostras examinadas foram incluídas no herbário do Departamento de Botânica da Universidade Federal de Minas Gerais (BHCB).

\section{Resultados e Discussão}

No presente trabalho foram identificados 28 táxons específicos e infra-específicos, distribuídos entre as divisões Cyanobacteria, Euglenophyta, Pyrrophyta (Cryptophyceae e Dinophyceae) e Chrysophyta (Chrysophyceae). Dentre eles, 23 são ocorrências novas para o estado de Minas Gerais. As diatomáceas (Bacillariophyceae) são pobremente representadas nessa represa, sendo apenas uma espécie (Aulacoseira granulata var. angustissima) presente em maiores densidades e, portanto, não foram incluídas nessa descrição.

A seguir encontram-se as descrições e ilustrações dos táxons identificados.

\section{EUGLENOPHYTA}

Euglenophyceae

Euglenales

Euglenaceae

Euglena oxyuris Schmarda

Figura 1.

Células pouco metabólicas, alongadas, em fita, um pouco achatadas, geralmente espiraladas. Cauda curta. Membrana ornada de estrias helicoidais bem marcadas. Cromatóforos numerosos e discóides, sem pirenóides. Anéis de paramilo na frente e atrás do núcleo. Flagelo geralmente de comprimento igual a metade do comprimento da célula. Compr. 140-210 $\mu \mathrm{m}$, larg. 14-22 $\mu \mathrm{m}$.

Distribuição geográfica no estado de Minas Gerais: primeira citação da espécie.

\section{Lepocinclis salina f. pachyderma Deflandre}

Figura 2.

Células ovóides em vista frontal, pólos arredondados, pólo anterior atenuado, assimétrico, com uma depressão oblíqua que vai até o centro da célula, pólo posterior amplamente arredondado, mais largo. Película hialina a amarelo-claro, bastante espessa, estriada, em hélice, estrias dextrógiras. Cloroplastos numerosos, discóides, parietais. Grãos de paramilo numerosos, alongados ou arredondados. Estigma grande. Comprimento do flagelo quase duas vezes maior que o da célula, emergindo lateralmente. Compr. 25,1-30,1 $\mu \mathrm{m}$, larg. 18,4$24,2 \mu \mathrm{m}$.

Distribuição geográfica no Brasil: primeira citação da forma.

\section{Lepocinclis salina var. vallicauda Conrad}

Figura 3.

Células ovóides em vista frontal, arredondadas ou ligeiramente elípticas, pólo anterior arredondado, assimétrico, pólo posterior terminando abruptamente em uma cauda cônica, arredondada e oblíqua. Película incolor ou pouco colorida, amarelada, estrias dextrógiras. Cloroplastos numerosos, discóides, parietais. Grãos de paramilo numerosos, parietais, arredondados a elípticos. Estigma volumoso. Flagelo geralmente de comprimento 1-3 vezes maior que o da célula, emergindo lateralmente. Compr. 28,5-60 $\mu \mathrm{m}$, larg. 21,7-36,7 $\mu \mathrm{m}$.

Distribuição geográfica no estado de Minas Gerais: primeira citação da espécie e da variedade.

\section{Phacus longicauda Ehrenberg}

Figura 4.

Células de contorno arredondado ou elíptico, mais ou menos simétricas; parte posterior prolongando-se em uma cauda bem comprida, reta. Película grossa, rígida e com estrias longitudinais bem marcadas, desde o ápice até a cauda, raramente torcidas em hélice. Cloroplastos numerosos, discóides, de posição parietal, sem pirenóides. Grão de paramilo 
em forma de anel e outros pequenos dispersos no protoplasma. Compr. 54-63 mm, com a cauda 153 $161 \mu \mathrm{m}$, larg. 38-44 $\mu \mathrm{m}$.

Distribuição geográfica no estado de Minas Gerais: primeira citação da espécie.

Phacus onyx Pochmann

Figura 5.

Células com contorno arredondado e trapezoidal, assimétricas; um dos lados, ou os dois, curvados de forma característica; parte posterior terminando abruptamente. Cauda oblíqua e fortemente curvada. Película com estrias longitudinais. Cloroplastos numerosos, discóides, sem pirenóides. Grão de paramilo 1 , central, às vezes um segundo, menor. Compr. 21-29 $\mu \mathrm{m}$, larg. 18-25 $\mu \mathrm{m}$.

Distribuição geográfica no estado de Minas Gerais: primeira citação da espécie.

Phacus tortus (Lemm.) Skvortzov

Figura 6.

Células levemente torcidas em parafuso (cerca de uma volta), simétricas em relação ao eixo central; parte posterior ligeiramente inchada, prolongada numa longa cauda reta ou levemente curvada. Película com estrias seguindo a torsão. Cloroplastos numerosos, discóides, sem pirenóides. Grânulos de paramilo 1-2 em forma de anel. Flagelo aproximadamente do tamanho do corpo. Compr. com a cauda 74-80 $\mu \mathrm{m}$, larg. 32-46 $\mu \mathrm{m}$.

Distribuição geográfica no estado de Minas Gerais: município de Belo Horizonte, reservatório da Pampulha (Giani \& Leonardo 1988).

Trachelomonas acanthostoma var. minor Drezepolski

Figura 7.

Lórica elíptica, finamente pontuada ou granulada. Poro largo, rodeado por pequenos espinhos. Compr. 19,2-21,7 $\mu \mathrm{m}$, larg. 16,7-19,2 $\mu \mathrm{m}$.

Distribuição geográfica no Brasil: primeira citação da espécie.

Trachelomonas armata (Ehrenberg) Stein

Figura 8.

Lórica hialina até marrom-amarelada muito escura, elipsoidal a levemente ovóide, lisa ou com freqüência finamente e densamente pontuada, pólos arredondados, o anterior às vezes um pouco estreito, o posterior com uma coroa de espinhos fortes, recurvados, convergentes, de comprimento variável, às vezes reduzidos a simples papilas curtas. Poro com ou sem espessamento anular, às vezes provido de um colo baixo, denticulado-crenulado. Compr. 31-35 $\mu \mathrm{m}$, larg. 25-29,3 $\mu \mathrm{m}$.

Distribuição geográfica no estado de Minas Gerais: município de Belo Horizonte, reservatório da Pampulha (Giani \& Leonardo 1988).

\section{Trachelomonas curta Da Cunha}

Figuras 9-10.

Lórica marrom-escura, elipsoidal, lisa, os dois pólos curvados de forma idêntica. Poro simples ou munidos de uma protuberância anular. Flagelos 3-4 vezes o comprimento do corpo. Compr. 8,3-11 $\mu \mathrm{m}$, larg. $10-12 \mu \mathrm{m}$.

Comentário: O material estudado apresenta tamanho menor do que o normalmente descrito na literatura, mas assemelha-se às dimensões registradas por Menezes (1994).

Distribuição geográfica no estado de Minas Gerais: primeira citação da espécie.

Trachelomonas verrucosa var. granulosa Playfair Figuras 11-12.

Lórica marrom-amarelada até vermelho-escura, esférica ou ligeiramente subesférica, coberta por granulações serreadas, quase hemisféricas, rodeada por pequenas verrugas, muito numerosas. Poro de borda expandida em anel. Diâm. 15-16 $\mu \mathrm{m}$.

Comentário: O tamanho observado é menor do que o normalmente citado para a espécie tipo.

Distribuição geográfica no estado de Minas Gerais: primeira citação da variedade.

Trachelomonas verrucosa f. irregularis Deflandre Figura 13.

Lórica espessa, de marrom-amarelada até vermelho-escura, esférica ou ligeiramente subesférica com numerosas granulações, de tamanho irregular, não muito serreadas. Diâm. 14-15 $\mu \mathrm{m}$.

Distribuição geográfica no estado de Minas Gerais: primeira citação da forma.

Trachelomonas volvocina Ehrenberg

Figuras 14-16.

Lórica de cor amarela ou marrom, esférica, lisa. Poro anterior com espessamento anular pouco proe- 
minente, às vezes com um colo muito baixo. Dois cromatóforos com pirenoídes. Flagelos 2-3 vezes o comprimento do corpo. Diâm. 10-17 $\mu \mathrm{m}$.

Distribuição geográfica no estado de Minas Gerais: Brejo da Lapa, Parque Nacional do Itatiaia (Bicudo \& Ventrice 1968).

\section{Trachelomonas volvocinopsis Swirenko}

Figura 17.

Lórica marrom, esférica, lisa. Células com 10 cromatóforos discóides, desprovidos de pirenóides. Flagelo longo, cerca de 3 vezes o comprimento do corpo. Diâm. 17-20 $\mu \mathrm{m}$.

Comentário: As células desta espécie diferem de T. volvocina apenas pelo número de cromatóforos e ausência de pirenóides.

Distribuição geográfica no estado de Minas Gerais: primeira citação da espécie.

\section{PYRROPHYTA}

Cryptophyceae

Cryptomonadales

Cryptomonadaceae

Cryptomonas brasiliensis Castro, Bicudo \& Bicudo Figuras 18-19.

Célula em vista lateral de contorno sub-sigmóide, margem dorsal assimétrica, amplamente convexa nos $2 / 3$ anteriores, margem ventral uniformemente convexa, pólo anterior obliquamente truncado, às vezes porção dorsal proeminente formando rostro pouco evidente, pólo posterior afilado, agudo, voltado para a face dorsal da célula. Cromatóforos 2, laterais. Pirenóides 2 ou 4, 1-2 por cromatóforo. Numerosos grãos de amido, elípticos ou quase, esparsos. Citofaringe pouco a bem evidente, 0,2 a 0,5 do comprimento da célula. Ejectissômios globosos, pouco visíveis. Corpúsculos de Maupa não observados. Flagelos 2, subapicais, de tamanho pouco distinto entre si, aproximadamente tão longos quanto a célula. Compr. 12-23 $\mu \mathrm{m}$, larg. 6,7-13,5 $\mu \mathrm{m}, \mathrm{Rc} / 1=$ 1,5-2,1.

Distribuição geográfica no estado de Minas Gerais: primeira citação da espécie.

Cryptomonas curvata Ehrenberg emend. Penard Figuras 20-22.

Célula em vista lateral de contorno subsigmóide, alongada, margem dorsal assimétrica, levemente convexa nos $2 / 3$ anteriores, margem ventral pouco convexa, quase reta, pólo anterior obliquamente truncado, porção dorsal proeminente formando rostro evidente, pólo posterior afilado, obtuso ou arredondado, voltado para a face dorsal da célula. Cromatóforos 2 , dorsiventrais. Pirenóides ausentes, às vezes numerosos grãos de amido elípticos ou quase, esparsos. Citofaringe desde pouco até evidente, 0,2-0,3 do comprimento da célula. Ejectissômios globosos, em várias camadas circundando a citofaringe. Córpusculo de Maupa 1. Flagelos 2, subapicais, tamanho pouco distinto entre si, ca. 0,5 do comprimento da célula. Compr. 30,1-45(-52) $\mu \mathrm{m}$, larg. 12,5-18,4 $\mu \mathrm{m}$, $\mathrm{Rc} / 1=1,9-2,6$.

Distribuição geográfica no estado de Minas Gerais: primeira citação da espécie.

\section{Cryptomonas erosa Ehrenberg}

Figuras 23-25.

Célula com vista lateral de contorno elíptico, oblongo a obovado, margem dorsal regular a irregularmente convexa, margem ventral convexa, reta ou levemente côncava, raro irregularmente convexa, pólo anterior obliquamente truncado, porção dorsal proeminente formando rostro desde pouco a bastante evidente, às vezes sem rostro, pólo posterior arredondado, às vezes afilado. Cromatóforos 2 , dorsiventrais. Pirenóides ausentes, às vezes numerosos grãos de amido elípticos, esparsos. Citofaringe pouco a muito evidente, 0,3-0,7 do comprimento da célula. Ejectissômios globosos ou naviculóides, em várias camadas, circundando a citofaringe. Corpúsculos de Maupa não observados. Flagelos 2, subapicais, tamanho pouco distinto entre si, aproximadamente tão longos quanto a célula. Compr. 14,2-31,5 $\mu \mathrm{m}$, larg. 9,2-15 $\mu \mathrm{m}, \mathrm{Rc} / 1$ $=1,6-2,1(-3,1)$.

Distribuição geográfica no estado de Minas Gerais: primeira citação da espécie.

\section{Rhodomonas lacustris Pascher \& Ruttner}

Figuras 26-28.

Células subovóides, curvadas e piriformes quando vistas lateralmente, em vistas frontal e dorsal apresentam-se piriformes, mas não curvadas lateralmente. Pólo anterior obliquamente truncado. Pólo posterior curvado e fortemente pontudo. Pirenóide 1, proeminente, embebido no cromatóforo, presente no lado dorso-ventral. Ejectissômios usualmente não visíveis. Corpúsculos de Maupa não observados. Flagelos 
2, do tamanho da célula ou mais curtos, inseridos no pólo anterior oblíquo. Compr. 8,4-11,7 $\mu \mathrm{m}$, larg. 4,2-6,8 $\mu \mathrm{m}, \mathrm{Rc} / 1=1,5-2,4$.

Comentário: Javornicky (1976) descreve R. minuta Skuja e outras Rhodomonas, como R. lacustris e $R$. lens e sugere a sinonimização destas espécies. Os nossos espécimes conferem, pela sua morfologia e tamanho, tanto com a descrição de $R$. lacustris sensu Castro et al. (1991) quanto com a de R. minuta var. nannoplanctonica em Rojo \& Alvarez Cobelas (1993). Resolvemos, no momento, adotar a classificação usada por Castro et al. (1991), tanto por se tratar de um estudo realizado com material brasileiro, quanto por estar de acordo com a sinonímia das espécies proposta por Javornicky (1976).

Distribuição geográfica no estado de Minas Gerais: primeira citação da espécie.

Dinophyceae

Peridiniales

Peridiniaceae

Peridinium volzii Lemmermann

Figuras 29-31.

Células esféricas, um pouco achatadas ventralmente, freqüentemente mais largas que longas, parte inferior da célula um pouco menor que a superior. Sulco transversal helicoidal, sulco longitudinal prolongando-se na epiteca. Placas areoladas. Tabulação da epiteca: 7 placas postequatoriais ("), 4 apicais (') e 3 intermediárias (a); da hipoteca: 5 postequatoriais ("') e 2 anti-apicais ("”). Compr. 41,8-52,6 $\mu$ m, larg. 33,4-41,8 $\mu \mathrm{m}, \mathrm{Rc} / 1=1,1-1,3$.

Comentário - Esta espécie diferencia-se de $P$. willei, por não apresentar uma "crista" (ou "pente") localizada na margem da placa apical ou nas margens do sulco transversal.

Distribuição geográfica no estado de Minas Gerais: município de Belo Horizonte, reservatório da Pampulha (Giani \& Leonardo 1988).

\section{CHRYSOPHYTA}

Chrysophyceae

Chromulinales

Mallomonadaceae

Mallomonas producta var. marchica Lemm.

Figura 32.

Células elíptico-alongadas até quase cilíndricas, arredondadas nos pólos, às vezes um pou- co convexas. Escamas rômbicas, em fileiras transversais a diagonais. Setas retas ou ligeiramente curvadas, lisas, do tamanho do corpo. Cromatóforos 2, laterais, parietais, marrom-dourados. Compr. da célula sem setas 21,7-35,1 $\mu \mathrm{m}$, larg. $10-16,7 \mu \mathrm{m}$.

Comentário: Esta variedade difere da variedade tipo por: tamanho menor, células um pouco mais curtas e relativamente mais largas e setas às vezes presentes nos dois pólos (Smith 1920).

Distribuição geográfica no Brasil: primeira citação da variedade.

Rhizochrysidiales

Rhizochrysidiaceae

Rhizochrysis schefferlii Pascher

Figura 33.

Células em pequenos agrupamentos, arredondadas, com inúmeros (10-12) rizópodos delicados, que às vezes se ramificam. Vacúolo contrátil presente. Cromatóforos 1-2, grandes. Cistos arredondados. Diâm. 18-21(-30) $\mu \mathrm{m}$.

Distribuição geográfica no Brasil: primeira citação da espécie.

\section{CYANOBACTERIA}

Cyanophyceae

Chroococcales

Microcystaceae

Microcystis flos-aquae (Wittr.) Kirchner

Figuras 42-43, 45.

Colônias esféricas ou elipsoidais quando jovens, geralmente não lobadas, mas às vezes o contorno externo é irregular e quando velhas podem aparecer cavidades indistintas. Bainha mucilaginosa fina, incolor, formando uma margem bem fina em volta das células. Células esféricas, densa e homogeneamente aglomeradas. Numerosos aerotopos presentes. Diâm. $(2,1-) 2,5-2,9(-3,2) \mu \mathrm{m}$.

Comentário - Segundo Komárek (1991) esta espécie foi freqüentemente identificada como uma forma de $M$. aeruginosa. Ele afirma, porém, nunca ter encontrado forma de transição entre as duas espécies. A densidade das células na colônia, a forma da colônia e o diâmetro das células são características que diferenciam $M$. flos-aquae de $M$. aeruginosa.

Distribuição geográfica no estado de Minas Gerais: primeira citação da espécie. 

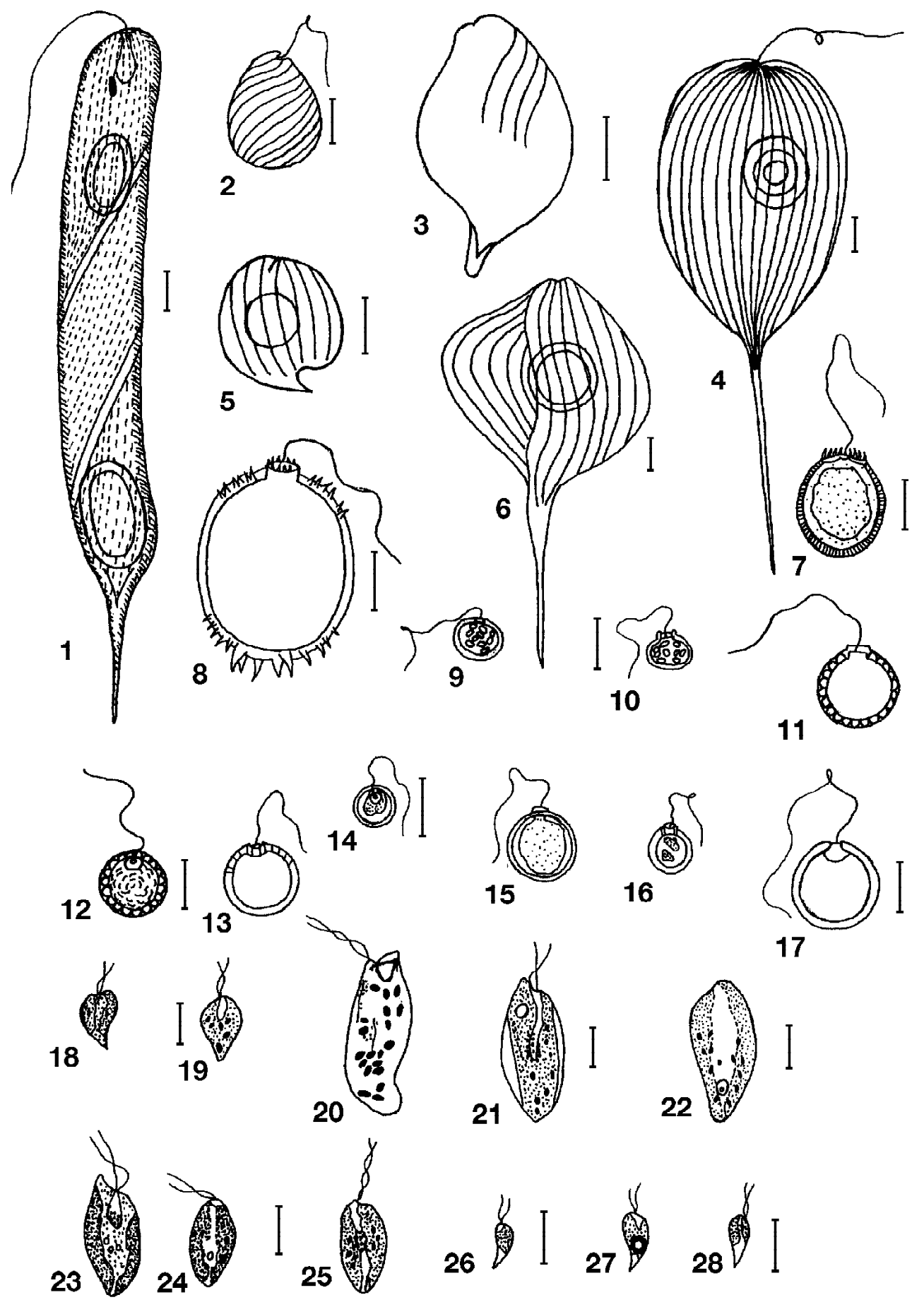

Figura 1. Euglena oxyuris. Figura 2. Lepocinclis salina f. pachyderma. Figura 3. L. salina var. vallicauda. Figura 4. Phacus longicauda. Figura 5. P. onyx. Figura 6. P. tortus. Figura 7. Trachelomonas acanthostoma var. minor Figura 8. T. armata. Figuras 9-10. T. curta. Figuras 11-12. T. verrucosa var. granulosa. Figura 13. T. verrucosa f. irregularis. Figuras 14-16. T. volvocina. Figura 17. T. volvocinopsis. Figuras 18-19. Cryptomonas brasiliensis. Figuras 20-22. C. curvata. Figuras 23-25. C. erosa. Figuras 26-28. Rhodomonas lacustris. Escalas $=10 \mu \mathrm{m}$. 

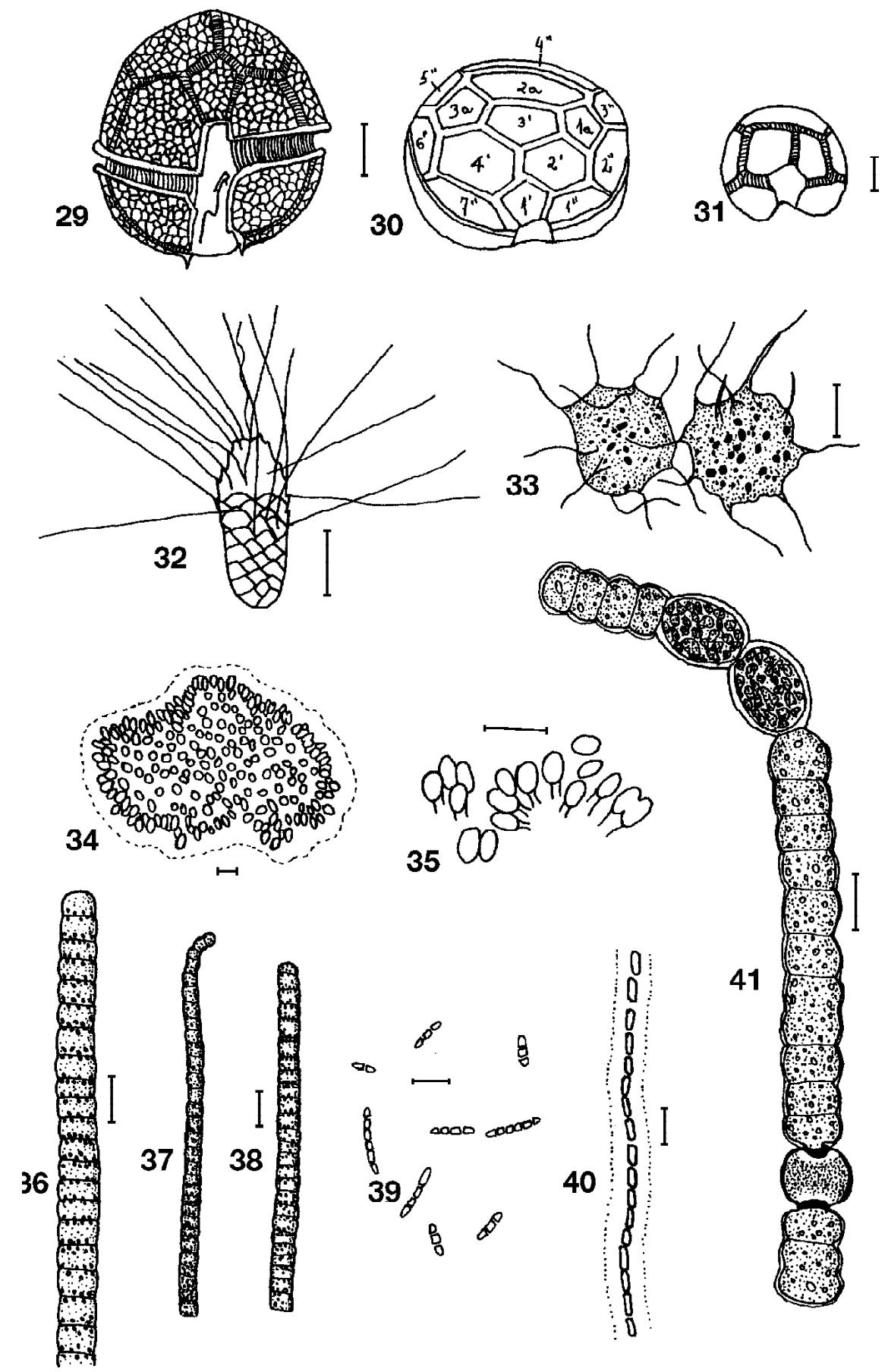

Figuras 29-31. Peridinium volzii. Figura 32. Mallomonas producta var. marchica. Figura 33. Rhizochrysis schefferlii. Figuras 34-35. Woronichinia naegeliana. Figura 36. Phormidium tenue. Figuras 37-38. Phormidium cf. tenue. Figura 39. Pseudanabaena mucicola. Figura 40. P. catenata. Figura 41. Anabaena solitaria. Escalas $=10 \mu \mathrm{m}$. 

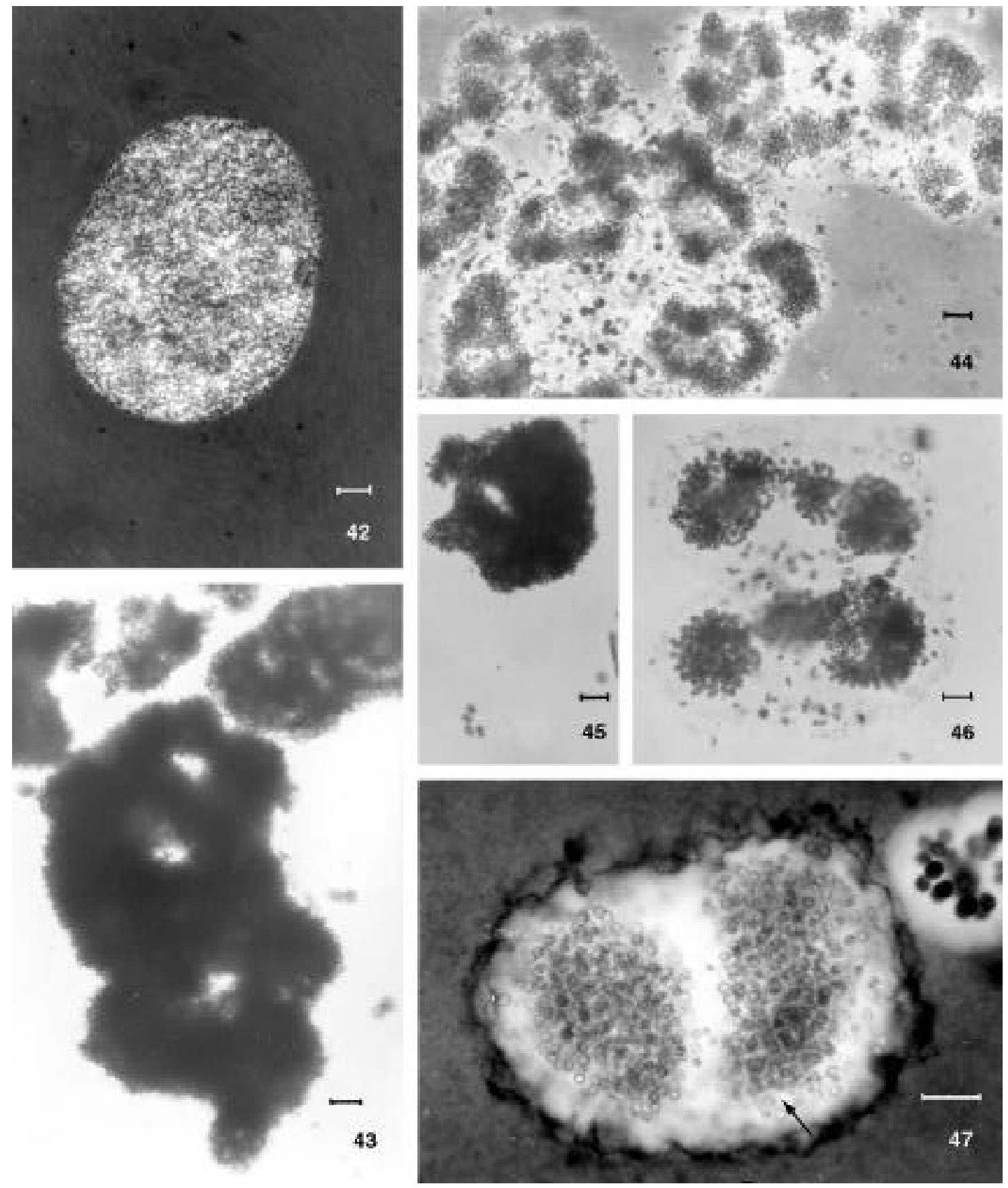

Figuras 42-43, 45. Microcystis flos-aquae. Figuras 44, 46-47. M. viridis. Escalas $=20 \mu \mathrm{m}$ 
Microcystis viridis (A. Br.) Lemmermann

Figuras 44, 46-47.

Colônias redondas ou retangulares, consistindo de um grande número de colônias filhas aglomeradas em conjuntos irregulares, colocados em planos diferentes, mas sem cavidades distintas. Colônias envolvidas por uma bainha mucilaginosa comum, ampla e refrativa, margens da bainha colonial bem definidas. Células esféricas, numerosos aerotopos presentes. Diâm. (3,2-)3,8-5,5 $\mu \mathrm{m}$.

Distribuição geográfica no estado de Minas Gerais: primeira citação da espécie.

\section{Woronichinia naegeliana (Unger) Elenkin}

Figuras 34-35.

Colônia esférica, elipsoidal ou irregular. Células ovais ou elipsoidais, densamente arranjadas, com orientação radiada na periferia da colônia, unidas por pedúnculos largos e radiais de mucilagem. Aerotopos presentes. Compr. 5-6 $\mu \mathrm{m}$, larg. 2,5-4 $\mu \mathrm{m}$.

Distribuição geográfica no estado de Minas Gerais: primeira citação da espécie.

Oscillatoriales

Phormidiaceae

Phormidium tenue (Agardh ex Gomont) Anagnostidis \& Komárek

Figura 36.

Tricomas solitários, retos, não atenuados em direção ao ápice, constritos. Células 1,3-2,2 vezes mais largas que longas, conteúdo celular azul-esverdeado, septos transversais granulados, célula apical arredondada. Compr. 4,2-5,5 $\mu \mathrm{m}$, larg. 6,7-10 $\mu \mathrm{m}$.

Comentário: A espécie tem geralmente como habitat a região litorânea de lagos e reservatórios, mais do que o plâncton. É possível que seu aparecimento no plâncton do reservatório da Pampulha seja causado por despreendimento dos filamentos da região litorânea.

Distribuição geográfica no estado de Minas Gerais: Brejo da Lapa, Parque Nacional do Itatiaia (Bicudo \& Ventrice 1968: como Oscillatoria tenuis).

Phormidium cf. tenue (Agardh ex Gomont) Anagnostidis \& Komárek

Figuras 37-38.

Tricomas solitários, retos ou curvados no ápice, não atenuados, constritos. Células (0,8-) 1,0-1,7(-2,0) vezes mais largas que longas, conteúdo celular azulesverdeado, septos transversais granulados, célula apical arredondada ou cilíndrico-arredondada. Compr. 2,5-3,7(-4,2) $\mu \mathrm{m}$, larg. 3,3-5 $\mu \mathrm{m}$.

Comentários: Esta população assemelha-se muito ao material descrito por Sant'Anna \& Azevedo (1995) como Oscillatoria cf. tenuis Agardh ex Gomont. Da mesma forma, difere da espécie-tipo apenas pelo menor tamanho de suas células.

Distribuição geográfica no estado de Minas Gerais: primeira citação da espécie.

Pseudanabaenaceae

Pseudanabaena mucicola (Naumann \& HuberPestalozzi) Schwabe

Figura 39.

Tricomas curtos, 2-6 células, sempre presos à mucilagem de algas planctônicas. Células cilíndricas ou aproximadamente isodiamétricas, azul-esverdeadas, célula apical cilíndrico-cônica. Aerotopos ausentes. Compr. 2,5-4,2(-5,0) $\mu \mathrm{m}$, larg. 1,7-2 $\mu \mathrm{m}$.

Comentário: No reservatório da Pampulha esta espécie sempre foi observada nas bainhas das espécies de Microcystis.

Distribuição geográfica no estado de Minas Gerais: primeira citação da espécie.

\section{Pseudanabaena catenata Lauterborn}

Figura 40.

Tricomas flutuando livremente, às vezes envolvidos por bainha mucilaginosa. Células cilíndricas, truncadas. Aerotopos ausentes. Compr. $5 \mu \mathrm{m}$, larg. $2 \mu \mathrm{m}$.

Distribuição geográfica no estado de Minas Gerais: primeira citação da espécie.

Nostocales

Nostocaceae

Anabaena solitaria Klebahn

Figura 41.

Tricomas retos ou ligeiramente curvados, solitários, flutuantes, bainha mucilaginosa pouco desenvolvida. Células esféricas a elipsóides. Aerotopos presentes. Heterocito esférico, da mesma largura dos filamentos. Acineto cilíndrico, distante do heterocito, quando maduro mais largo que o filamento. Célula: compr. (5,0-)7,3-8 $\mu \mathrm{m}$, larg. 7,5-11 $\mu \mathrm{m}$. Heterocito:

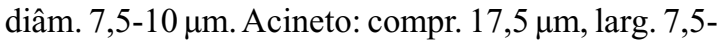
$12 \mu \mathrm{m}$.

Distribuição geográfica no estado de Minas Gerais: primeira citação da espécie. 
O grande número de citações novas para o estado de Minas Gerais ( $82 \%$ dos táxons descritos neste trabalho), mostra como trabalhos ficoflorísticos devem ser incentivados na região. Na divisão Cyanobacteria, $88 \%$ das oito espécies identificadas são primeiras citações para esse estado. Para Euglenophyta, 77\% dos 13 táxons identificados são registrados pela primeira vez. Todas as espécies das classes Cryptophyceae e Chrysophyceae são primeiras ocorrências no estado.

Além disso, diversas espécies aqui identificadas, como algumas criptofíceas e euglenofíceas, ainda apresentam distribuição bastante restrita no Brasil como conseqüência do menor número de trabalhos realizados com estes grupos. Por causa disto, registramos quatro táxons como primeira ocorrência no Brasil: Lepocinclis salina f. pachyderma e Trachelomonas acanthostoma var. minor (Euglenophyta) e Mallomonas producta var. marchica e Rhizochrysis schefferlii (Chrysophyta).

Agradecimentos - Às Dras. Célia L. Sant'Anna, Maria Tereza P. Azevedo e Miriam B. Xavier, pela colaboração nas identificações dos táxons descritos; à equipe (presente e passada) do projeto de "Monitoramento limnológico do reservatório da Pampulha" pela coleta das amostras. O projeto foi financiado através de bolsa de I.C. do CNPq para C.C. Figueredo e do PRPq/UFMG para P.C. Eterovick.

\section{Referências bibliográficas}

ANAGNOSTIDIS, K. \& KOMÁREK, J. 1988. Modern approach of classification system of Cyanophytes. 3- Oscillatoriales. Arch. Hydrobiol. Suppl. 80:327-472.

ALVES-DA-SILVA, S.M. \& LAITANO, C.S. 1994. Euglenaceae pigmentadas do Banhado do Jacaré, em um parque de proteção ambiental, Triunfo, RGS, Brasil. Iheringia 45:89-116.

ARAUJO, M.A.R. 1995. Produção e consumo de carbono orgânico na comunidade planctônica da Represa da Pampulha, Belo Horizonte, MG. Dissertação de mestrado, Universidade Federal de Minas Gerais, Belo Horizonte.

BALECH, E. 1944. Trachelomonas de la Argentina. An. Mus. Argent. Cienc. Nat., Buenos Aires 41:233-305.

BICUDO, C.E.M. \& VENTRICE, M.R. 1968. Algas do Brejo da Lapa, Parque Nacional do Itatiaia, Brasil. In Anais do XIX Congresso da Sociedade de Botânica do Brasil, p. 3-42.

CASTRO, A.A.J., BICUDO, C.E.M. \& BICUDO, D.C. 1991. Criptógamos do Parque Estadual das Fontes do Ipiranga, São Paulo, SP. Algas, 2: Cryptophyceae. Hoehnea 18:87-106.

CONRAD, W. \& VAN MEEL, L. 1952. Materiaux pour une monographie de Trachelomonas Ehrenberg, C., 1834, Strombomonas Deflandre, G., 1930, et Euglena Ehrenberg, C. 1832, genres d'Euglenacées. Inst. Roy. Sc. Nat. Belg. Bruxelles 124:1-176.

DEFLANDRE, G. 1926. Monographie du genre Trachelomonas Ehr. Nemours, Paris.
DESIKACHARY, T.V. 1959. Cyanophyta. Indian Council of Agricultural Research, New Delhi.

ETEROVICK, P.C. \& GIANI, A. 1997. Levantamento taxonômico das Chlorophyta (Volvocales, Chlorococcales, Zygnematales) do Reservatório da Pampulha, MG. Revta brasil. Bot. 20:79-90.

GIANI, A. \& LEONARDO, I.M. 1988. Distribuição vertical de algas fitoplanctônicas no Reservatório da Pampulha, Belo Horizonte, MG. Acta Limnol. Brasil. 2:387-404.

GOODWIN, K.L. 1997. Dinâmica das populações de Cyanobacteria no Reservatório da Pampulha (MG) em duas escalas temporais (sazonal e diurna). Dissertação de mestrado, Universidade Federal de Minas Gerais, Belo Horizonte.

HUBER-PESTALOZZI, G. 1962. Das Phytoplankton des Süsswassers. Systematik und Biologie. 2. Teil. 1. Hälfte. Chrysophyceeen, farblose Flagellaten, Heterokonten. E. Schweizerbart'sche Verlagsbuchhandlung, Stuttgart.

HUBER-PESTALOZZI, G. 1969. Das Phytoplankton des Süsswassers. Systematik und Biologie. 4. Teil. Euglenophyceen. E. Schweizerbart'sche Verlagsbuchhandlung, Stuttgart.

JAVORNICKY, P. 1976. Minute species of the genus Rhodomonas Karsten (Cryptophyceae). Arch. Protistenkd. 118:98-106.

KOMÁREK, J. 1991. A review of water-bloom forming Microcystis species, with regard to population from Japan. Algol. Stud. 64:115-127.

KOMÁRKÓVA-LEGNEROVÁ, J. \& ELORANTA, P. 1992. Planktic blue-green algae (Cyanophyta) from Central Finnland (Jyväskyla region) with special reference to the genus Anabaena. Algol. Stud. 67:103-133.

MENEZES, M. 1994. Fitoflagelados pigmentados de quatro corpos d'água da região sul do município do Rio de Janeiro, Brasil. Tese de doutorado, Universidade de São Paulo, São Paulo.

PARRA, O.O., GONÇALES, M., DELLAROSSA, V., RIVIERA, P. \& ORELLANA, M. 1982. Manual taxonomico del fitoplancton de aguas continentales, com referencia al plancton de Chile. III. Cryptophyceae, Dinophyceae, Euglenophyceae. Ed. Universidad de Concepción, Concepción.

PINTO-COELHO, R.M., GIANI, A. \& VON SPERLING, E. 1994. Ecology and human impact on lakes and reservoirs in Minas Gerais. SEGRAC, Belo Horizonte.

POCHMANN, A. 1942. Synopsis der Gattung Phacus. Arch. Protistenkd. 95:81-252.

PORTER, K.G. 1989. Phagotrophic phytophlagellates in microbial food webs. Hydrobiologia 159:89-97.

ROJO, C. \& ALVAREZ-COBELAS, M. 1993. Taxonomy and ecology of phytoplankton in a hypertrophic, gravel-pit lake. II. Cryptophyceae, Euglenophyceae, Dinophyceae. Nova Hedwigia 57:47-63.

SANT'ANNA, C.L. \& AZEVEDO, M.T.P. 1995. Oscillatoriaceae (Cyanophyceae) from São Paulo State, Brazil. Nova Hedwigia 60:19-58.

SCHILLER, J. 1937. Dinoflagellatae (Peridinae) in monographisches Behandlung. In Rabenhorst's Kryptogamen-Flora von Deutschland, Österreich und der Scweiz. 10, Abt. 3, 2 Teil. Akademische Verlagsgesellschaft, Leipzig. p.1-390.

SMITH, G.M. 1920. Phytoplankton of the inland lakes of Wisconsin. Part I. Myxophyceae, Phaeophyceae, Heterokontae, and Chlorophyceae exclusive of the Desmidiaceae. Wisc. Geol. Nat. hist. Bull. 57:1-243.

XAVIER, M.B. 1989. O gênero Lepocinclis Perty de Lagos do Parque Estadual das Fontes do Ipiranga, São Paulo, Brasil. Hoehnea 16:133-147. 